Article

\title{
Novel Remanence Determination for Power Transformers Based on Magnetizing Inductance Measurements
}

\author{
Chen Wei ${ }^{1, *}$, Xianqiang $\mathrm{Li}^{1}$, Ming Yang ${ }^{2}$, Zhiyuan $\mathrm{Ma}^{1}$ and Hui Hou ${ }^{1}$ \\ 1 School of Automation, Wuhan University of Technology, Wuhan 430070, China; lxq@whut.edu.cn (X.L.); \\ mazhiyuan@whut.edu.cn (Z.M.); houhui@whut.edu.cn (H.H.) \\ 2 School of Electrical Engineering, Chongqing University, Chongqing 400044, China; cqucee@cqu.edu.cn \\ * Correspondence: lucius_wei@whut.edu.cn
}

Received: 16 October 2019; Accepted: 2 December 2019; Published: 5 December 2019

\begin{abstract}
The remanence (residual flux) in the core of power transformers needs to be determined in advance to eliminate the inrush current during the process of re-energization. In this paper, a novel method is proposed to determine the residual flux based on the relationship between residual flux and the measured magnetizing inductance. The paper shows physical, numerical, and analytical explanations on the phenomenon that the magnetizing inductance decreases with the increase of residual flux under low excitation. Numerical simulations are performed by EMTP (Electro-Magnetic Transient Program) on a $1 \mathrm{kVA}$ power transformer under different amounts of residual flux. The inductance-remanence curves are nearly the same when testing current changes. Laboratory experiments conducted on the same transformer are in line with the numerical simulations. Furthermore, numerical simulation results on a 240 MVA are reported to demonstrate the effectiveness of the proposed method.
\end{abstract}

Keywords: magnetizing inductance; power transformer; residual flux

\section{Introduction}

Power transformer is the key device of electrical energy system, the performance of transformers directly affects the stability of the power system. When a power transformer is de-energized, there is always a certain amount of remanence (residual flux) left in the iron core due to the hysteresis of the ferromagnetic material [1-4]. This residual flux may cause negative effects when re-energizing the transformer, such as inrush currents, overvoltages, dynamic stresses, and resonances. In addition, the inrush currents caused by residual flux can even result in relay protection misoperation. Time-controlled switching is an effective method to eliminate inrush currents [5-7]. For the method to work, it is essential to know the residual flux.

To determinate the residual flux of transformers, several methods have been proposed in recent years [8-16]. In a transformer factory, the residual flux is usually estimated from experience. According to the survey of International Council on Large Electric Systems in 1984, the result shows that only two in all 500 transformers tested have the residual flux of more than 0.7 times the amount of rated flux [8]. It is generally believed that the residual flux is $20 \%$ to $80 \%$ of the saturation flux [9].

Numeral research results on the prediction of residual flux mainly rely on the magnetic core mathematical model. Common hysteresis models such as Stoner-Wohlfarth (S-W) model, Jiles-Atherton (J-A) model [10], and Preisach model [3] have been applied. Although the prediction result of the mathematical method is satisfying, to estimate the residual flux, a series of material-related parameters are needed before simulations. Furthermore, the accuracy of prediction results depends 
largely on the accuracy of the selected parameters which cannot be detected directly. Moreover, the above prediction methods take too much time and are not suitable for industrial applications.

One applied method is based on the aging characteristics of magnetic material and Preisach model. The magnetic flux in the iron core is obtained from different segments of the excitation current's phase when the transformer is de-energized [11]. Since the fact that the inductive component current cannot be abruptly changed to zero, there is an error between the residual flux and the magnetic flux at the moment of de-energization. In addition, the detection equipment is expensive and complicated to operate.

D. Cavallera et al. employ a method based on the measurements using fluxgate sensors of the magnetic field induced by the leakage flux around the transformer [12]. The critical points for this method are the leakage flux measurement quality for small values (near zero) and the distance between the sensor and the magnetic circuit. The advantage of this method is its real-time nature. To obtain the real-time residual flux, its accuracy depends on the sensitivity and location of sensors. Additionally, this method costs too much and only suitable for transformers without tanks.

Yacamini and Abu-Nasser calculate the residual flux from the inrush current waveform during the process of the re-energization of a transformer [13]. This method is not feasible for field measurements because the value of the residual flux is obtained after the re-energization of the transformer, which defeats the purpose.

$\mathrm{Li}$ et al. directly measure residual flux in a small toroidal transformer by putting a gauss meter into the pre-cut air gap. The flux density in the air gap is considered to be the flux density of the iron core because the gap length could be ignored compared to the whole magnetic length [14]. The gauss meter is a more efficient way to measure the remanence in the core directly. However, the tested transformer must have an air gap and have no tanks. It is an advantageous experimental approach instead of an industrial detection means.

Another widely used method to determinate the residual flux is voltage integration during the de-energization of transformers [14-16]. In [14], the residual flux is determined by the voltage integral when numerical simulations performed on a $220 \mathrm{~V} / 11 \mathrm{~V}, 50 \mathrm{~Hz}, 8 \mathrm{VA}$ small toroidal transformer. In [15], phasor and amplitude method are chosen to determine the time limit of integration. In [16], in order to evaluate the actual influence of system transients on the residual flux, the remanence is approximated by the voltage integral at the transformer terminal and core phenomena have been measured with a frequency response analyzer. The shortcoming of this method is its inaccuracy due to low-voltage signals mixed with dc component and noise. In addition, it is difficult to determine the initial time for integration.

Baris Kovan and Francisco de León propose a technique to mitigate large inrush currents in network transformers by reducing the residual flux in the core of transformers. The aim of reduction of residual flux is achieved by using a low-power ultra-low frequency voltage source. This source produces a single-phase rectangular voltage waveform [17]. The variable voltage constant frequency (VVCF) and the variable frequency constant voltage (VFCV) are compared. The variable frequency constant voltage (VFCV) performs better than the other one. The results show it reduces the residual flux in the core successfully. However, the key to this method is to determinate the amount and direction of residual flux.

In view of the shortcomings of the above methods, a method for accurately and quickly measuring the residual flux is needed. Ideally, the method can be implemented in real-time in the field measurement. The magnetization process of the core is divided into reversible domain wall displacement, irreversible domain wall displacement, and reversible domain wall rotation. In [18], the author points out that all the irreversible changes in domains lead to the hysteresis. Moreover, the domain structure rarely undergoes irreversible changes with low excitations whether the material contains remanence or not. That means a small signal excitation causes little residual flux. Thus, a novel technique to determine the residual flux is proposed in this paper based on measurements of the magnetizing inductance using a small signal excitation. In summary, the proposed method can be done by the following steps. First, the 
relationship between the residual flux and the magnetizing inductance of the transformer needs to be obtained in the factory in advance. By performing an open-circuit experiment, the remanence-flux curve can be drawn. Second, the magnetizing inductance is measured with a low signal excitation before the transformer is re-energizing. Last, the newest remanence is determined directly by comparing the measured inductance and the remanence-inductance curve obtained before. The main advantage of this method is that it can be done conveniently before re-energizing the transformer. To validate the proposed method, simulations and laboratory experiments are performed on a $1 \mathrm{kVA}, 60 \mathrm{~Hz}, 240 \mathrm{~V} / 120$ $\mathrm{V}$ transformer with the small excitation range from $0.5 \%$ to $2 \%$ of the rated voltage. The agreement between simulations and experiments shows pretty good results.

This paper is organized as follows. Section 2 outlines the magnetization mechanism of transformers and gives a new explanation on the relationship between residual flux and magnetizing inductance under low signal excitation. Section 3 describes the validation of the proposed method with EMTP simulations and experiments. Laboratory experiments on a small-size transformer are discussed, which are in line with the simulation results. The simulations on a large power transformer are performed to further validate the proposed method, too. Section 4 discusses the results of the simulations on the small transformer and the large one. Finally, conclusions are summarized in Section 5.

\section{Theoretical Background}

\subsection{Magnetization Mechanism}

The domain theory is the most widely accepted theoretical basis for the study of hysteresis characteristics [19]. Under the external magnetic field, the magnetic domains of ferromagnetic material gradually shift from the original random arrangement state to the state along the direction of the magnetic field strength. The magnetic domain rotation and the domain wall displacement occur, which changes the original magnetic domain structure. The material changes from a magnetic neutral state to a magnetic saturation state in which all magnetic domains have the same direction as the applied magnetic field. The magnetization process is shown in Figure 1.

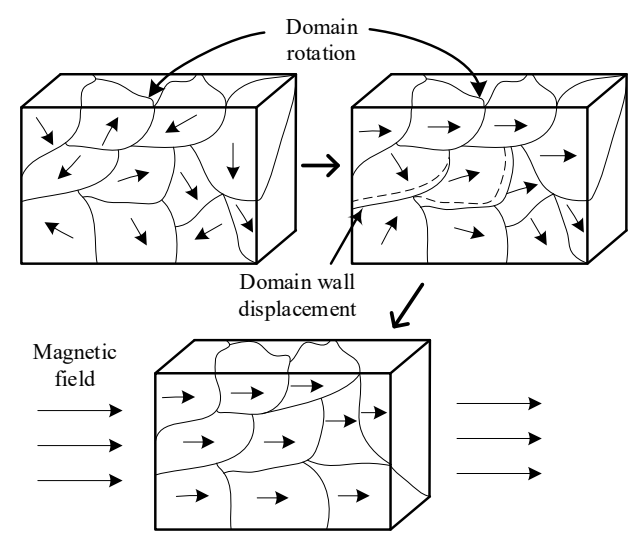

Figure 1. The process of magnetization.

The magnetization curves of most ferromagnetic materials show that the process from a magnetic neutral state to a magnetic saturation state includes domain wall displacement and magnetic domain rotation $[18,19]$. In a general situation, the magnetization process is generally divided into three stages. When external magnetic field is small enough, reversible domain wall displacement is the main structural change of the magnetic domain. With the enhancement of the external magnetic field, the Barkhausen jump happens. In this stage, irreversible domain wall displacement plays an important role. Moreover, hysteresis loss mainly occurs in this stage. In reversible domain wall rotation stage, the coil is behaving as if it is becoming an air-core [19-21]. The magnetic domain structure curve of different magnetization stages is shown in Figure 2. 


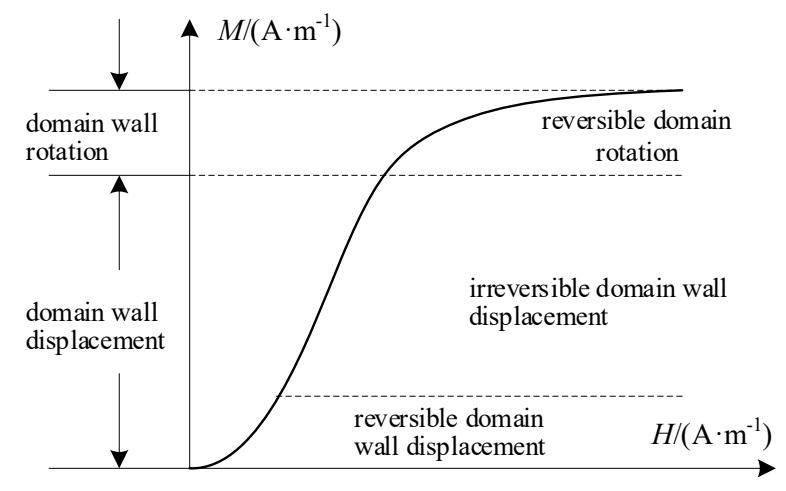

Figure 2. The magnetic domain structure of different magnetization stages.

The irreversible nonlinear response of the magnetization to an imposed magnetic field is the essential characteristic of any ferromagnetic material. Due to the irreversibility of the magnetization process, when the external magnetic field is removed, the magnetic flux density cannot follow the magnetic field strength down to zero, and there is a time lag of the magnetic flux density behind the magnetic field strength [22], which is the hysteresis, as shown in Figure 3a. Hysteresis is a property of ferromagnetic materials.

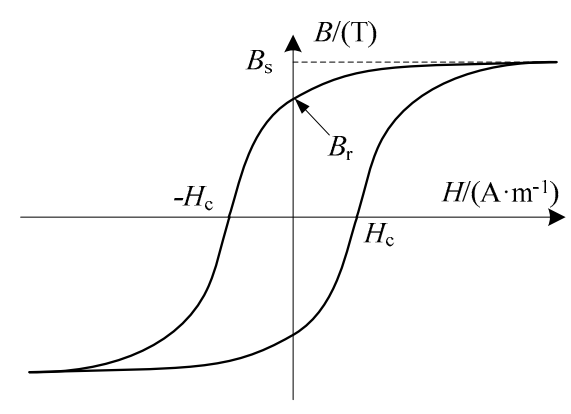

(a)

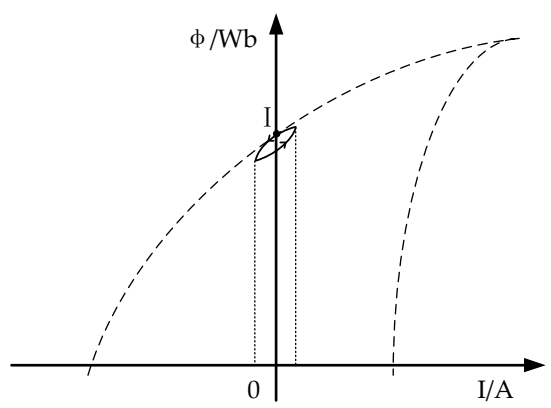

(b)

Figure 3. Hysteresis loop (a) under rated voltage; (b) under small excitation with remanence.

Due to the inherent hysteresis of the magnetic material, when the external magnetic field is reduced to zero, the magnetic material does not return to the magnetic neutral state but maintains a stable magnetization, that is, remanence. Remanence is a sign of irreversible magnetization and an important physical parameter that determines the shape of the hysteresis loop. When a small excitation is energizing the transformer, a path corresponding to the initial residual flux will be traced out (see Figure $3 b$ ). The residual flux will trace back to the initial status when de-energizing [23]. Based on the inherent hysteresis characteristic of the magnetic material, a small AC signal can be chosen to measure the magnetizing inductance with little effects on the residual flux.

\subsection{Relationship between Residual Flux and Inductance}

The residual flux only exists in the iron core. As a result, the residual flux influences the measured magnetizing inductance [24]. When the residual flux in the iron core is small enough, the transformer is working in the unsaturated period. The transformers running at rated voltage satisfy the following equations. Assuming that the rated voltage is $u$, which can be computed as follows:

$$
u=\sqrt{2} U \sin (\omega t+\alpha)
$$


The flux created by the magnetizing inductor is calculated as follows:

$$
\varphi_{L}=-\sqrt{2} \frac{U}{\omega} \cos (\omega t+\alpha)
$$

The flux in the core with residual flux is calculated as follows:

$$
\lambda=\Phi_{\text {res }}-\sqrt{2} \frac{U}{\omega} \cos (\omega t+\alpha)
$$

The real-time current satisfies the following equation:

$$
\begin{gathered}
i_{0}=\frac{\Phi_{\mathrm{res}}}{L_{\mathrm{ms}}}-\sqrt{2} \frac{U}{\omega L_{\mathrm{ms}}} \cos (\omega t+\alpha) \\
I_{0}=\frac{U}{\omega L_{\mathrm{ms}}} \sqrt{1+\left(\omega \Phi_{\mathrm{res}} / U\right)^{2}}
\end{gathered}
$$

where, $L_{\mathrm{ms}}$ is the measured magnetizing inductance without residual flux, $\Phi_{\text {res }}$ is the residual flux [25]. Therefore, the magnetizing inductance $L_{\mathrm{m}}$ is calculated as

$$
L_{\mathrm{m}}=\frac{U}{\omega I_{0}}=\frac{L_{\mathrm{ms}}}{\sqrt{1+\left(\omega \Phi_{\mathrm{res}} / U\right)^{2}}}
$$

A conclusion can be drawn based on Equation (6): Due to the existence of residual flux, the measured magnetizing inductance reduces to $1 / \sqrt{1+\left(\omega \Phi_{\text {res }} / U\right)^{2}}$ times of the magnetizing inductance without residual flux. In other words, the magnetizing inductance is decreasing with the enhancement of residual flux.

To further investigate the mechanism of the phenomenon that the higher the residual flux the smaller magnetizing inductance is, the following inference from the perspective of domain theory is conducted.

According to the magnetization mechanism described before, the hysteresis loop is formed because the magnetic domain wall displacement and the magnetic domain rotation occur in the internal magnetic domain of the ferromagnetic material under the external magnetic field [18]. If there is residual flux in the iron core, it means that the applied magnetic field is sufficiently large in the previous magnetization process. The coupling strength between domains has changed and the iron core underwent the stage of irreversible domain wall displacement.

Then, if a sufficiently small magnetic field is imposed on the iron core with residual flux, it causes the ferromagnetic material to undergo mainly the reversible domain wall displacement. The original domain that has undergone irreversible domain wall displacement will not participate in the re-magnetization process. That is to say, when a sufficiently small signal is applied, only a part of the magnetic domain participates in the re-magnetization process for the iron core in which the residual flux is present.

For the magnetizing inductor, when the core is not saturated, the inductance can be calculated as

$$
L=\frac{\mu_{r} \mu_{0} N^{2} S}{l_{m}}
$$

where, $\mu_{r}$ is the relative permeability of the iron core, $\mu_{0}$ is the vacuum permeability, $N$ is the number of the turns of the coil, $S$ is the cross-sectional area of the iron core, and $l_{m}$ is the average magnetic path length. The path length and the cross-sectional area of an iron core without excitation are presented in Figure 4. 


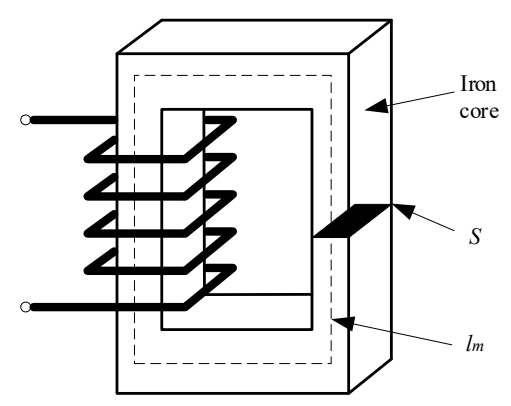

Figure 4. Magnetic path length and the cross-sectional area of the core.

The domains that participate in the magnetization process can be divided into several groups:

(a) Those undergoing reversible domain wall displacement;

(b) Those that have accomplished irreversible domain wall displacement;

(c) Those that have finished reversible domain rotation.

With the enhancement of external magnetic field, more and more domains get through the irreversible change process. The domain wall structure is becoming stable. Those domains which cannot be easily changed by low excitation perform as an air core, or a hypothetical totally magnetized part. Domains in group (b) and (c) cannot be easily changed under low excitation. In the unsaturated state, a hypothetical totally magnetized part (comprises domains in group (b) and (c)) and a hypothetical partly magnetized part compose the whole iron core. As shown in Figure 5, the inner dark part of the iron core is equivalent to the hypothetical totally magnetized part, which can be regarded as a saturated core. In addition, the magnetic inductance is proportional to the magnetic permeability. When the excitation imposed to the primary coil is small enough, the change of magnetic permeability is not obvious. In this paper, the change in permeability caused by the small signal applied during the test is assumed to be small enough. The magnetizing inductance and the magnetic flux density in the detecting coil satisfy the following equation:

$$
L=\frac{\mu N^{2} S_{e}}{l_{m}}
$$

where $S_{e}$ is the cross-sectional area of hypothetical partly magnetized core. This part can be further magnetized with low excitation, the cross-sectional area of which is defined as effective cross-sectional area of the core in this paper. It can be seen from Equation (8) that the inductance of the core inductor is proportional to the effective cross-sectional area of the core. As the strength of the external magnetic field increases, the effective cross-sectional area decreases. Apparently, the measured inductance declines, too.

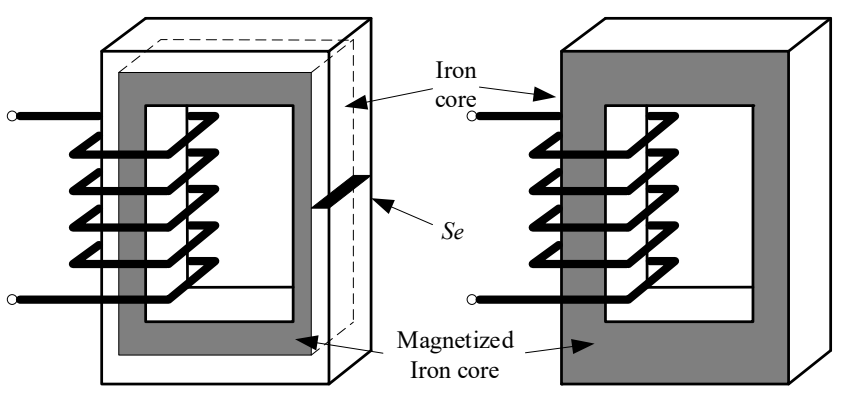

(a)

(b)

Figure 5. Iron core with (a) partial magnetization (b) saturation.

The inference in this section shows physical, numerical, and analytical explanations on why the measured inductance decreases with the increase of residual flux. When there is residual flux in the 
iron core, it can be considered that the effective cross-sectional area of the core becomes smaller. Thus, the inductance of the inductor with residual flux will decrease. Under low excitation, the reversible domain wall displacement mainly occurs, and the loss during the magnetization process is almost negligible. The magnetic flux density can be returning to the origin along the strength of the magnetic field, the peak value of the magnetizing current in the winding of the closed magnetic core depends on the initial value of residual flux [5]. Therefore, before the transformer is re-energized due to a fault trip or planned maintenance, the residual flux can be determined based on measuring the magnetizing inductance of the transformer using a sufficiently small signal.

\section{Simulations and Experiments Results}

\subsection{Numerical Simulations}

In order to validate the effectiveness and feasibility of the proposed method, simulations are performed using a $1 \mathrm{kVA}$ laboratory transformer (rated at $240 \mathrm{~V} / 120 \mathrm{~V}$ ) on the EMTP platform.

The arrangement of the simulations is illustrated in Figure 6. Additionally, the values of the simulation settings are given in Table $1 . R_{1}$ represents the short-circuit impedance of the source. The hysteresis characteristic of the transformer is modeled with the built-in hysteresis fitter in the EMTP platform. The core model is usually based on the open-circuit transformer test measurement. By the piecewise nonlinear characteristic, the data of the $\lambda_{m}-i_{m}$ magnetizing curve (the relationship of the flux linkage and the current) of the transformer is measured and presented in Table 2 [26]. $R_{2}$ represents the eddy loss of the iron core. The nonlinear inductor with hysteresis and the resistance representing the eddy current loss constitute the excitation circuit of the transformer.

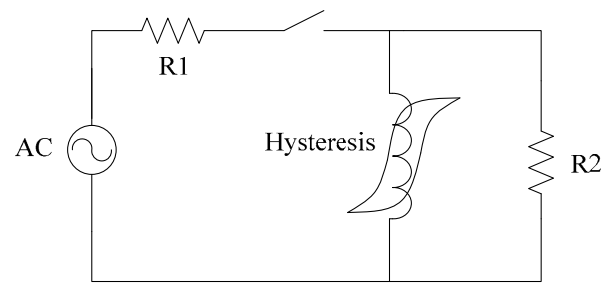

Figure 6. Simulation model.

Table 1. Parameters of the circuit of Figure 6.

\begin{tabular}{ccccccc}
\hline Elements & Frequency & Voltage Ratio & Capacity & Test Voltage & R1 & R2 \\
\hline Value & $60 \mathrm{~Hz}$ & $240 \mathrm{~V} / 120 \mathrm{~V}$ & $1 \mathrm{kVA}$ & $1 \mathrm{~V}-5 \mathrm{~V}$ & $1 \Omega$ & $1074.7 \Omega$ \\
\hline
\end{tabular}

Table 2. Magnetizing curve of the transformer (Referred to low side).

\begin{tabular}{cccccc}
\hline Point & $\boldsymbol{i}_{\boldsymbol{m}}(\mathrm{A})$ & $\boldsymbol{\lambda}_{\boldsymbol{m}} \mathbf{( W b )}$ & Point & $\boldsymbol{i}_{\boldsymbol{m}}(\mathbf{A})$ & $\left.\boldsymbol{\lambda}_{\boldsymbol{m}} \mathbf{( W b}\right)$ \\
\hline 1 & 0.078 & 0.0415 & 9 & 1.640 & 0.3870 \\
2 & 0.118 & 0.0880 & 10 & 2.086 & 0.4065 \\
3 & 0.160 & 0.1340 & 11 & 2.662 & 0.4250 \\
4 & 0.214 & 0.1780 & 12 & 3.792 & 0.4505 \\
5 & 0.300 & 0.2215 & 13 & 5.058 & 0.4705 \\
6 & 0.544 & 0.2760 & 14 & 6.880 & 0.4925 \\
7 & 0.816 & 0.3160 & 15 & 12.384 & 0.5415 \\
8 & 1.308 & 0.3655 & - & - & - \\
\hline
\end{tabular}

In the transformer low-voltage short circuit impedance test and winding frequency response test, the voltage $V_{\text {rms }}$ and current $I_{\text {rms }}$ of the transformer excitation branch are measured, and the magnetizing inductance is calculated as 


$$
L=V_{\mathrm{rms}} /\left(\omega I_{\mathrm{rms}}\right)
$$

To minimize the influence of the applied signal on the residual flux, a small signal ranging from 1 to $5 \mathrm{~V}$ is used which generates a current range from 5.3 to $26.5 \mathrm{~mA}$.

Figures 7 and 8 are the magnetic flux curve when the residual flux is $0.1 \mathrm{~Wb}$ and the applied voltage is 1 and $5 \mathrm{~V}$, respectively. In Figures 7 and 8, b is the partial zoomed in figure a. One can appreciate that there is a flux offset due to the residual flux, and this flux offset gradually decreases. However, the amount of flux offset reduction is very small within $10 \mathrm{~s}$. When the applied voltage is 1 and $5 \mathrm{~V}$, respectively, the flux offset is reduced by approximately $1 \%$ and $4 \%$ in $10 \mathrm{~s}$. In this case, the current $I_{\mathrm{rms}}$ of the transformer excitation branch is about 5.3 and $26.5 \mathrm{~mA}$ (see Figure 9), which is $0.064 \%$ and $0.32 \%$ of the rated current of the transformer, respectively. Based on the discussions in Section 2, the imposed current is very small and it has neglected influence on the residual flux.

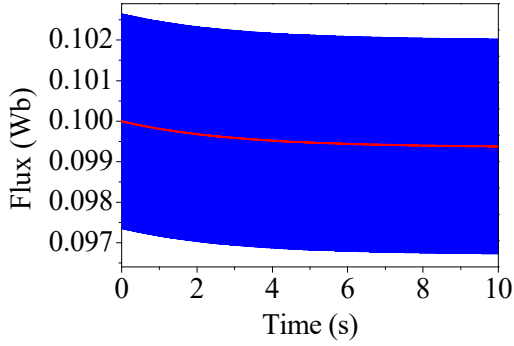

(a)

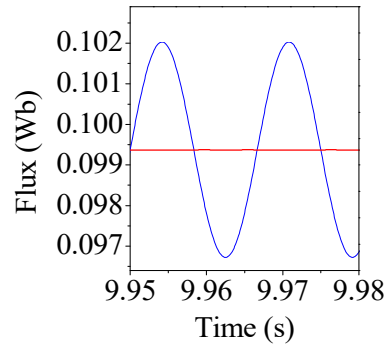

(b)

Figure 7. The flux curve under $1 \mathrm{~V}$ : (a) Flux curve within $10 \mathrm{~s}$; (b) partial zoomed in.

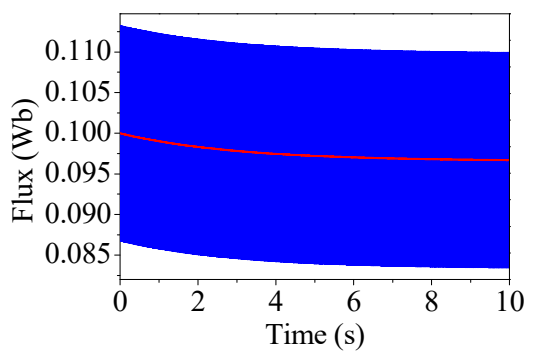

(a)



(b)

Figure 8. The flux curve under $5 \mathrm{~V}$ : (a) Flux curve within $10 \mathrm{~s}$; (b) partial zoomed in.

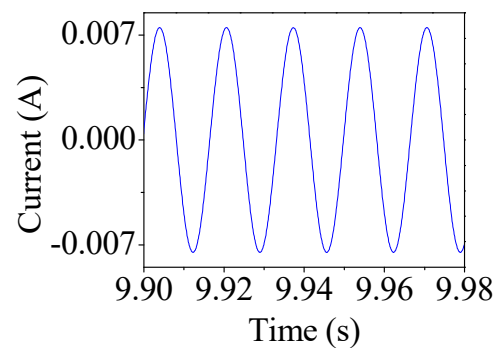

(a)

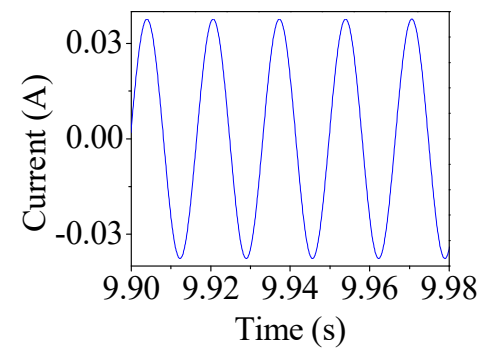

(b)

Figure 9. The test current: (a) Test voltage $1 \mathrm{~V}$; (b) test voltage $5 \mathrm{~V}$.

The simulation results of magnetizing inductance of the transformer under different residual flux when the test voltage is $1 \mathrm{~V}$ are shown in Figure 10. One can conclude the tendency is very clear that the magnetizing inductance becomes smaller with the increase of residual flux in the transformer core. 




Figure 10. The inductance-remanence curve under $1 \mathrm{~V}$ test voltage.

As shown in Figure 10, the magnetizing inductance of the transformer is $0.3533 \mathrm{H}$, when there is no residual flux in the iron core. If the residual flux is increased to $0.125 \mathrm{~Wb}$, the magnetizing inductance is reduced by $6.34 \%$ to $0.3309 \mathrm{H}$.

Table 3 presents the results of the magnetizing inductance under different residual flux when the test voltage changes from 2 to $5 \mathrm{~V}$. The inductance-remanence curve under $1-5 \mathrm{~V}$ test voltage is shown in Figure 11. It can be seen that the variation of the magnetizing inductance with the change of residual flux is exactly the same as the case of $1 \mathrm{~V}$. When the residual flux increases from zero to $0.125 \mathrm{~Wb}$, the magnetizing inductance is reduced by about $6 \%$. However, in the case of the same residual flux, when the test voltage changes from 1 to $5 \mathrm{~V}$, the magnetizing inductance is basically unchanged. This shows that the magnetizing inductance is linear in the small signal region.

Table 3. Inductance under different residual and test voltage in simulations (H).

\begin{tabular}{ccccc}
\hline \multirow{2}{*}{$\begin{array}{c}\text { Residual Flux } \\
(\mathbf{W b})\end{array}$} & $\mathbf{4}$ & \multicolumn{4}{c}{ Test Voltage (V) } \\
\cline { 2 - 5 } & $\mathbf{2}$ & $\mathbf{3}$ & $\mathbf{4}$ & $\mathbf{5}$ \\
\hline $\mathbf{0}$ & 0.3532 & 0.3531 & 0.353 & 0.3528 \\
$\mathbf{0 . 0 2 5}$ & 0.3504 & 0.3504 & 0.3504 & 0.3504 \\
$\mathbf{0 . 0 5 0}$ & 0.3469 & 0.3469 & 0.3469 & 0.3469 \\
$\mathbf{0 . 0 7 5}$ & 0.3425 & 0.3426 & 0.3426 & 0.3426 \\
$\mathbf{0 . 1 0 0}$ & 0.3359 & 0.336 & 0.336 & 0.3361 \\
$\mathbf{0 . 1 2 5}$ & 0.3310 & 0.3312 & 0.3314 & 0.3315 \\
\hline
\end{tabular}

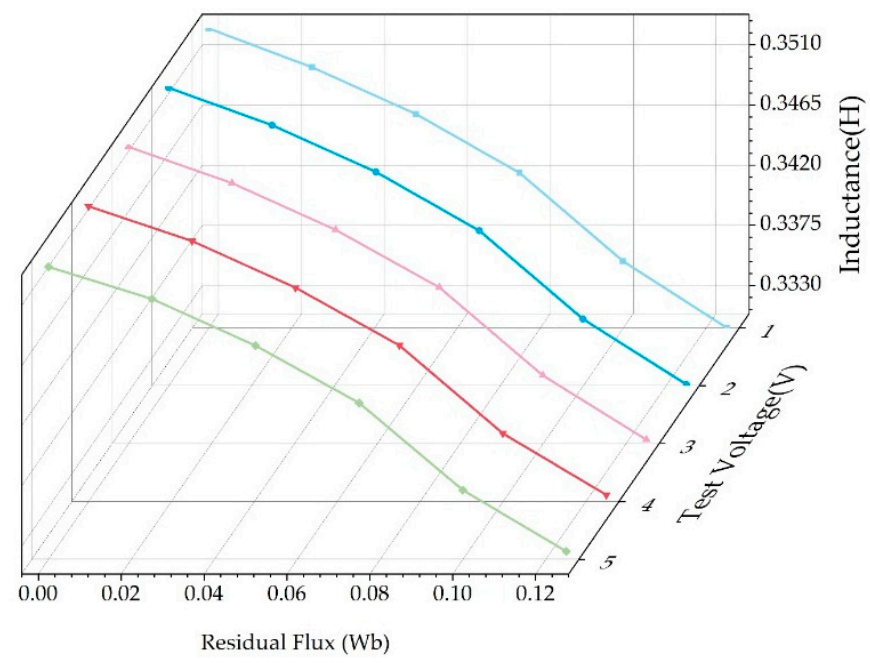

Figure 11. The inductance-remanence curve under 1-5 V test voltage. 


\subsection{Laboratory Experiments}

Laboratory experiments are conducted on a single-phase toroidal transformer which has the same parameters as the one in the simulations above. The parameters of the tested transformer are given in Table 1. Before each test, the transformer is demagnetized by energizing the transformer using an AC voltage regulator at a rated voltage and then gradually reducing the voltage to zero [27]. A DC source excites the transformer to a magnetized status, and then the DC source is disconnected to make the transformer have a residual flux. Then, the magnetizing inductance of the transformer is measured using a high-precision LCR meter (Inductance, Capacitance and Resistance meter). The setup of the experiment is shown in Figure 12.

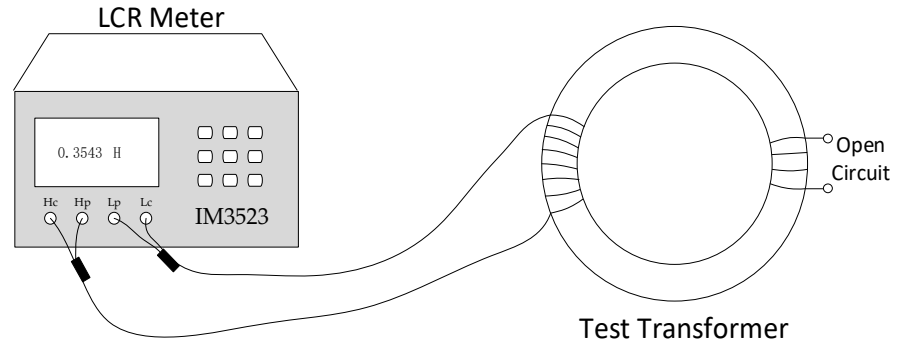

(a)

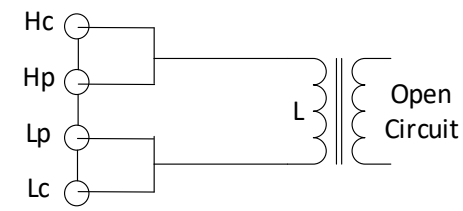

(b)

Figure 12. The laboratory setup of high-precision LCR meter (a) and the connection of the probe (b).

To ensure the measuring accuracy, the LCR meter must have the minimum test range of $0.1 \mathrm{mH}$. Furthermore, a measurement voltage of not more than $5 \mathrm{~V}$ is needed. The IM3523 LCR meter is chosen to measure the magnetizing inductance. More details about the meter is given in Table 4 .

Table 4. Parameters of the LCR meter.

\begin{tabular}{|c|c|}
\hline Measured Frequency & $40 \mathrm{~Hz}-200 \mathrm{kHz}$ \\
\hline Measuring Frequency & $1 \mathrm{kHz}$ \\
\hline Measurement Range & $10-100 \mathrm{M} \Omega$ \\
\hline Basic Accuracy & $\mathrm{Z}: \pm 0.05 \%$ rdg. $\theta: \pm 0.03^{\circ}$ \\
\hline Minimum Inductance & $1 \mu \mathrm{H}$ \\
\hline Test Voltage & $5 \mathrm{mV}-5 \mathrm{~V}$ \\
\hline
\end{tabular}

Table 5 shows the results of the measured magnetizing inductance from the experiments. One can see that as the excitation current increases, that is, the residual flux increases, the transformer magnetizing inductance becomes smaller. This trend is consistent with the simulation results in Figure 11. In the case of $15 \mathrm{~mA}$ test current, when the residual flux is zero, the magnetizing inductance is $0.3347 \mathrm{H}$. When the excitation current is increased to $4.98 \mathrm{~A}$, the magnetizing inductance is $0.3234 \mathrm{H}$, which is reduced by $3.38 \%$. Based on the basic accuracy of the LCR meter, the relative error of measurement is less than $0.05 \%$. The absolute error of the magnetizing inductance is not more than $150 \mu \mathrm{H}$.

\subsection{Simulations on a Large Power Transformer}

As can be seen from the previous simulations and experiments, although the tendency of the magnetizing inductance to decrease with the increase of residual flux is obvious, the change range of the magnetizing inductance is not large when the residual flux increases from zero to its maximum value. This is because the transformer used for the previous simulations and experiments is a small-size transformer, and the value of the magnetizing inductance is not large. 
Table 5. The measured inductance under laboratory experiments $(\mathrm{H})$.

\begin{tabular}{ccccc}
\hline \multirow{2}{*}{$\begin{array}{c}\text { Excitation Current } \\
\text { (A) }\end{array}$} & \multicolumn{4}{c}{ Test Current (mA) } \\
\cline { 2 - 5 } & $\mathbf{1 5}$ & $\mathbf{1 9}$ & $\mathbf{2 1}$ & $\mathbf{2 4}$ \\
\hline $\mathbf{0}$ & 0.3347 & 0.3311 & 0.3282 & 0.3255 \\
$\mathbf{0 . 0 8 1}$ & 0.3330 & 0.3294 & 0.3267 & 0.3240 \\
$\mathbf{0 . 1 2 8}$ & 0.3322 & 0.3287 & 0.3259 & 0.3233 \\
$\mathbf{0 . 4 4 4}$ & 0.3283 & 0.3254 & 0.3225 & 0.3200 \\
$\mathbf{1 . 4 6 0}$ & 0.3259 & 0.3230 & 0.3203 & 0.3177 \\
$\mathbf{4 . 9 8 0}$ & 0.3234 & 0.3209 & 0.3184 & 0.3158 \\
\hline
\end{tabular}

Theoretically, if it is a large power transformer, because its magnetizing inductance is relatively large, the variation of the magnetizing inductance should be relatively large when the residual flux changes between zero and maximum.

To validate this idea, simulations are performed on a large power transformer $(500 \mathrm{kV} / 240$ MVA). The parameters of the circuit in Figure 6 on the large power transformer are shown in Table 6 . The positive side of the large power transformer $\lambda_{m}-i_{m}$ characteristic (the relationship of the flux linkage and the current) is presented in Table 7 [28]. In simulations, the applied AC voltage is $100 \mathrm{~V}$, which generates a current less than $0.1 \%$ of the rated current of the transformer.

Table 6. Parameters of the circuit in Figure 6 on the large power transformer.

\begin{tabular}{ccccccc}
\hline Elements & Frequency & Voltage Ratio & Capacity & Test Voltage & R1 & R2 \\
\hline Value & $50 \mathrm{~Hz}$ & $500 \mathrm{kV} / 400 \mathrm{kV}$ & $240 \mathrm{MVA}$ & $100 \mathrm{~V}$ & $0.5 \Omega$ & $1526 \Omega$ \\
\hline
\end{tabular}

Table 7. Magnetizing curve of the $500 \mathrm{kV} / 240$ MVA transformer (Referred to high voltage side).

\begin{tabular}{cccccc}
\hline Point & $\boldsymbol{i}_{\boldsymbol{m}} \mathbf{( A )}$ & $\left.\boldsymbol{\lambda}_{\boldsymbol{m}} \mathbf{( W b}\right)$ & Point & $\boldsymbol{i}_{\boldsymbol{m}} \mathbf{( A )}$ & $\left.\boldsymbol{\lambda}_{\boldsymbol{m}} \mathbf{( W b}\right)$ \\
\hline 1 & 0.0016 & 4.17 & 20 & 0.2269 & 936.64 \\
2 & 0.0052 & 13.89 & 21 & 0.3357 & 1010.50 \\
3 & 0.0099 & 29.10 & 22 & 0.6009 & 1084.19 \\
4 & 0.0155 & 49.71 & 23 & 1.3337 & 1157.29 \\
5 & 0.0216 & 75.60 & 24 & 2.9336 & 1229.40 \\
6 & 0.0274 & 106.63 & 25 & 5.7142 & 1300.04 \\
7 & 0.0335 & 142.62 & 26 & 10.253 & 1368.95 \\
8 & 0.0393 & 183.37 & 27 & 16.154 & 1402.64 \\
9 & 0.0451 & 228.64 & 28 & 47.199 & 1436.16 \\
10 & 0.0510 & 278.18 & 29 & 107.010 & 1469.64 \\
11 & 0.0569 & 331.71 & 30 & 167.729 & 1502.55 \\
12 & 0.0630 & 388.91 & 31 & 227.671 & 1534.64 \\
13 & 0.0696 & 449.48 & 32 & 286.593 & 1566.00 \\
14 & 0.0775 & 513.06 & 33 & 343.885 & 1596.42 \\
15 & 0.0872 & 579.29 & 34 & 399.536 & 1625.93 \\
16 & 0.0990 & 647.79 & 35 & 453.556 & 1654.57 \\
17 & 0.1148 & 718.19 & 36 & 505.728 & 1682.18 \\
18 & 0.1360 & 790.06 & 37 & 555.828 & 1708.64 \\
19 & 0.1668 & 863.02 & 38 & 603.907 & 1734.09 \\
\hline
\end{tabular}

Figure 13 shows the inductance-remanence curve of the large power transformer obtained from the simulations. It can be seen that the magnetizing inductance decreases significantly with the increase of the residual flux. When there is no residual flux, the magnetizing inductance is $8.017 \mathrm{H}$; when the residual flux increases to $900 \mathrm{~Wb}$, the magnetizing inductance reduced to $1.021 \mathrm{H}$, which is reduced $87.3 \%$. 


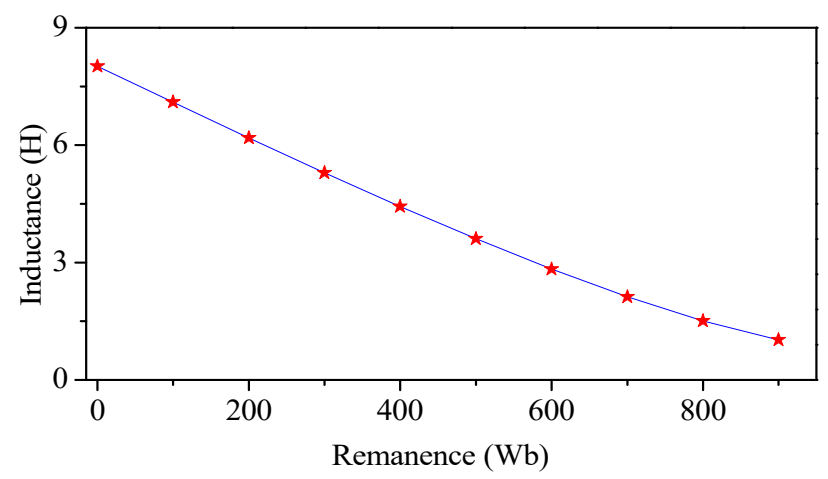

Figure 13. The inductance-remanence curve of the $500 \mathrm{kV} / 240 \mathrm{MVA}$ transformer.

\section{Discussion}

From the simulation results above, the inductance-remanence curves can be drawn by using low excitations on both small and large transformers. In this way, the residual flux can be determined through comparing the measured magnetizing inductance and the curves conveniently. In Figure 14, the two curves are compared for the fitting performance under the specified function. Since the change range of magnetizing inductance is different between the two kinds of transformers, a wider range of remanence-inductance curve can be measured on the large transformer. The large transformer shows a better fit for the liner function than the small one. Another key fact to remember, the sum squared residual of the large transformer is $7.69 \times 10^{-4}$ and it is $7.27 \times 10^{-6}$ on the small one, which means that the deviation can be ignored in both curves is small enough.

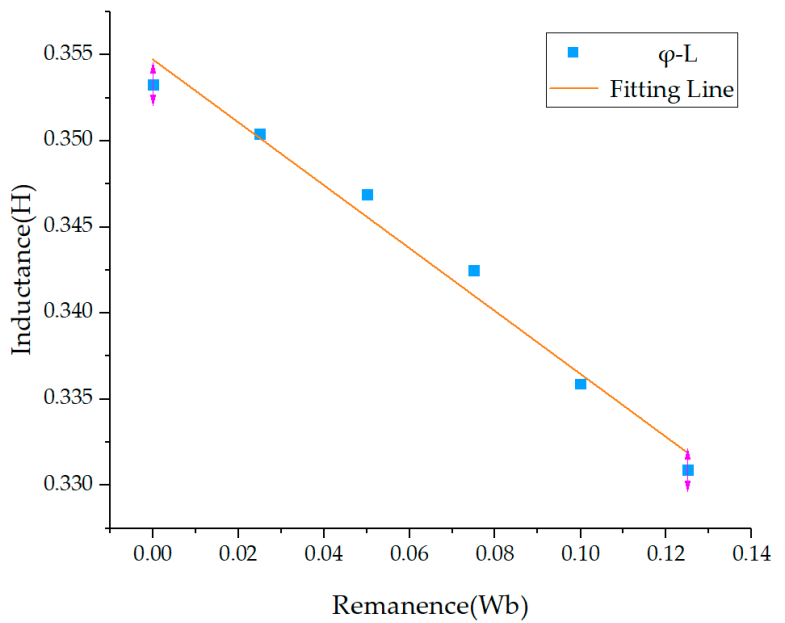

(a)

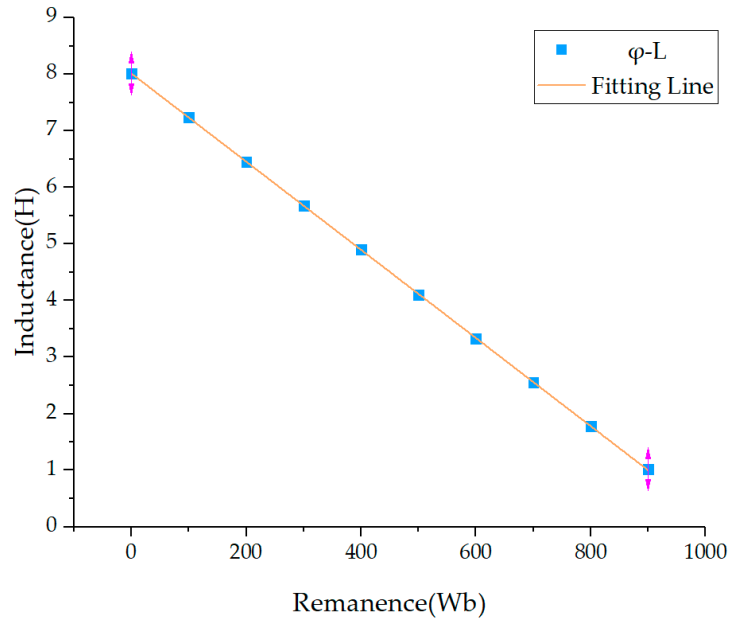

(b)

Figure 14. Comparison of (a) the $240 \mathrm{~V} / 1 \mathrm{kVA}$ transformer; (b) the $500 \mathrm{kV} / 240$ MVA transformer.

\section{Conclusions}

A novel method of the residual flux determination for power transformer based on the measurements of magnetizing inductance is proposed in this paper. This method can be done conveniently, and is especially suitable for on-site measurements. To avoid the influence of test current on residual flux, the test signal should be as small as possible. A test signal which is smaller than $0.1 \%$ of the rated current of the transformer is recommended. The relationship between the residual flux and the magnetizing inductance of the transformer can be determined in advance at the factory. The magnetizing inductance is measured with a small signal before the transformer re-energizes, and the value of the residual flux can be determined according to the obtained inductance-remanence curve. 
The performance of the proposed method is validated on a $1 \mathrm{kVA}, 60 \mathrm{~Hz}, 240 \mathrm{~V} / 120 \mathrm{~V}$ transformer for 1 to $5 \mathrm{~V}$ test voltage. The simulation results show that the remanence-inductance curve under low excitation is the same. The reason why the relationship between residual flux and magnetic inductance is the same is that low excitation has little effects on irreversible domain structure changes. Experimental tests on a transformer with the same parameters are in line with the numerical simulations. The simulations on a $500 \mathrm{kV} / 240 \mathrm{MVA}$ transformer further demonstrate that the proposed method is effective and feasible.

Author Contributions: All the authors contributed to publish this paper. Conceptualization, X.L. and C.W.; Data curation, Z.M.; Formal analysis, X.L. and C.W.; Methodology, X.L.; Supervision, X.L.; Validation, M.Y., X.L., and C.W.; Visualization, H.H. and Z.M.; Writing-Original Draft, X.L.; Writing-Review and Editing, C.W.

Funding: This research received no external funding.

Acknowledgments: The authors would like to express their gratitude to Francisco de León, of New York University, for his help and suggestions during the experimental stage and paper writing stage.

Conflicts of Interest: The authors declare no conflict of interest.

\section{References}

1. Jiles, D.C.; Atherton, D.L. Ferromagnetic hysteresis. IEEE Trans. Magn. 1983, 19, 2183-2185. [CrossRef]

2. Chwastek, K. Modelling offset minor hysteresis loops with the modified Jiles-Atherton description. J. Phys. D Appl. Phys. 2009, 42, 165002. [CrossRef]

3. Bertotti, G. Dynamic generalization of the scalar Preisach model of hysteresis. IEEE Trans. Magn. 1992, 28, 2599-2601. [CrossRef]

4. Stoner, E.C.; Wohlfarth, E.P. A mechanism of magnetic hysteresis in heterogeneous alloys. Trans. R. Soc. 1948, 240, 599-642. [CrossRef]

5. Chiesa, N.; Høidalen, H.K. Novel approach for reducing transformer inrush currents: Laboratory measurements, analytical interpretation and simulation studies. IEEE Trans. Power Deliv. 2010, 25, 2609-2616. [CrossRef]

6. Xing, Y.M.; Luo, J.; Zhou, J.P.; Xiong, Z.B.; Cai, M. Estimation of remanence in transformer core. Power Syst. Technol. 2011, 35, 169-172.

7. Wang, Y.; Liu, Z.Z.; Chen, H.X. Research on residual flux prediction of the transformer. IEEE Trans. Magn. 2017, 53, 6100304. [CrossRef]

8. Ge, W.Q. Simulation, Measurement and Demagnetization of the Residual Flux in the Iron Core of Power Transformer. Ph.D. Thesis, Hebei University of Technology, Tianjin, China, 2014.

9. Ge, W.Q.; Wang, Y.H.; Chen, X.G.; Xiao, S.X.; Yang, X.G.; Lv, D.N. Method to measure and weaken the residual flux of the power transformer core. Trans. China Electrotech. Soc. 2015, 30, 10-16.

10. Tao, F.B.; Zhang, G.; Li, J.S.; Wei, C.; Lu, Y.C.; Wu, P. Remanent magnetism estimation of transformer core based on local hysteresis loop slope. Transformer 2019, 56, 27-33.

11. Wu, Y.G.W. Analyze and Study of Control Strategy of Transformer Inrush Current. Ph.D. Thesis, Wuhan University, Wuhan, China, 2009.

12. Cavallera, D.; Oiring, V.; Coulomb, J.L.; Chadebec, O.; Caillault, B.; Zgainski, F. A new method to evaluate residual flux thanks to leakage flux, application to a transformer. IEEE Trans. Magn. 2014, 50, 7024904. [CrossRef]

13. Yacamini, R.; Abu-Nasser, A. Numerical calculation of inrush current in single phase transformers. IEE Proc. B Electr. Power Appl. 1998, 128, 327-334. [CrossRef]

14. Li, J.T.; Li, Z.; Li, T.; Gu, Y.; Jiang, W.; Zheng, M. Novel remanence detection for power transformers based on modified J-A model. In Proceedings of the TENCON 2015-2015 IEEE Region 10 Conference, Macao, China, 1-4 November 2015; pp. 1-3.

15. Yuan, W.Y.; Zhang, H.; Shangguan, Y.Q.; Zou, J.; Yuan, J.S. Analysis on method of calculating transformer residual flux by using the integration of port-voltage waveform and its implementation. In Proceedings of the 20th International Conference on Electrical Machines and Systems, Sydney, Australia, 11-14 August 2017; pp. $1-4$. 
16. Corrodi, Y.; Kamei, K.; Koyama, H.; Ito, H.; Goda, T. Influence of system transients on the residual flux of an unloaded transformer. In Proceedings of the IEEE Power Energy Society General Meeting, Detroit, MI, USA, 24-28 July 2011; pp. 1-7.

17. Kovan, B.; de Leon, F.; Czarkowski, D. Mitigation of inrush currents in network transformers by reducing the residual flux with an ultra-low-frequency power source. IEEE Trans. Power Deliv. 2011, 26, 1563-1570. [CrossRef]

18. Liorzou, F.; Phelps, B.; Atherton, D.L. Macroscopic models of magnetization. IEEE Trans. Magn. 2000, 36, 418-428. [CrossRef]

19. Yuan, D.F.; Ma, X.L. Magnetic Physics; University of Electronic Science and Technology Press: Chengdu, China, 1994; pp. 2-3.

20. Niu, S.J.; Zhao, L.H.; Chen, L.; Feng, Z.S.; Niu, C.C. Study on transformer residual flux detection method based on the time-current curve. Electr. Meas. Instrum. 2017, 54, 64-68.

21. Mclyman, C.W.T. Transformer and Inductor Design Handbook; China Electric Power Press: Beijing, China, 2013; pp. 153-164.

22. Zirka, S.E.; Moroz, Y.I.; Marketos, P.; Moses, A.J. A viscous-type dynamic hysteresis model as a tool for loss separation in conducting ferromagnetic laminations. IEEE Trans. Magn. 2005, 41, 1109-1111. [CrossRef]

23. Bruce, R.G.; Wright, A. Remanent flux in current-transformer cores. Proc. IEE 1966, 113, 915-920. [CrossRef]

24. Huang, Z.P. Calculation of Dynamic Inductance of Magnetic Core Coil, China Science and Technology Research Institute Annual Report (2008 Edition): Editorial Department of Science and Technology Annual Report; China Academy of Engineering Physics: Mianyang, China, 2009; Volume 2009.

25. Dou, Z.L.; Zhang, L.G.; Zhang, J.Z. Influence of transformer remanence on winding deformation test. Electr. Power Sci. Eng. 2017, 33, 54-60.

26. Yang, M.; Kazemi, R.; Jazebi, S.; Deswal, D.; de León, F. Retrofitting the BCTRAN transformer model with nonlinear magnetizing branches for the accurate study of low-frequency deep saturating transients. IEEE Trans. Power Deliv. 2018, 33, 2344-2353. [CrossRef]

27. De León, F.; Farazmand, A.; Joseph, P. Comparing the $\mathrm{T}$ and $\pi$ equivalent circuits for the calculation of transformer inrush currents. IEEE Trans. Power Deliv. 2012, 27, 2390-2398. [CrossRef]

28. Yang, M.; Deswal, D.; De Leon, F. Mitigation of half-cycle saturation of adjacent transformers during HVDC monopolar operation-Part II: Detecting zero-sequence fault currents. IEEE Trans. Power Deliv. 2019. [CrossRef] 